\title{
Modification of polyethylene glycol onto solid lipid nanoparticles encapsulating a novel chemotherapeutic agent (PK-L4) to enhance solubility for injection delivery
}

\author{
This article was published in the following Dove Press journal: \\ International Journal of Nanomedicine \\ 14 September 2012 \\ Number of times this article has been viewed
}

\author{
Yi-Ping Fang' \\ Pao-Chu Wu² \\ Yaw-Bin Huang ${ }^{3}$ \\ Cherng-Chyi Tzeng ${ }^{4}$ \\ Yeh-Long Chen ${ }^{4}$ \\ Yu-Han Hung ${ }^{2}$ \\ Ming-Jun Tsai ${ }^{5,6}$ \\ Yi-Hung Tsai ${ }^{3}$ \\ 'Department of Biotechnology, \\ Yuanpei University, Hsinchu, Taiwan; \\ ${ }^{2}$ School of Pharmacy, College of \\ Pharmacy, Kaohsiung Medical \\ University, Kaohsiung, Taiwan; \\ ${ }^{3}$ Graduate Institute of Clinical \\ Pharmacy, College of Pharmacy, \\ Kaohsiung Medical University, \\ Kaohsiung, Taiwan; ${ }^{4}$ School of \\ Medicinal and Applied Chemistry, \\ College of Life Science, Kaohsiung \\ Medical University, Kaohsiung, Taiwan; \\ ${ }^{5}$ Department of Neurology, China \\ Medical University Hospital, Taichung, \\ Taiwan; ${ }^{6}$ School of Medicine, Medical \\ College, China Medical University, \\ Taichung, Taiwan
}

Correspondence: Ming-Jun Tsai Department of Neurology, China Medical University Hospital, 2 Yuh-Der Road,

Taichung 404, Taiwan

Tel +886 422052 I 2 I ext 5039

Fax +886 422344055

Emaild22570@mail.cmuh.org.tw

Yi-Hung Tsai

Graduate Institute of Clinical Pharmacy, College of Pharmacy, Kaohsiung Medical University, 100 Shih-Chuan Ist Road,

Kaohsiung, 807 Taiwan

Tel +88673121101 ext 2166

Fax +88673210683

Email ypfang@mail.ypu.edu.tw
Background: The synthetic potential chemotherapeutic agent 3-Chloro-4-[(4-methoxyphenyl) amino]furo[2,3-b]quinoline (PK-L4) is an analog of amsacrine. The half-life of PK-L4 is longer than that of amsacrine; however, PK-L4 is difficult to dissolve in aqueous media, which is problematic for administration by intravenous injection.

Aims: To utilize solid lipid nanoparticles (SLNs) modified with polyethylene glycol (PEG) to improve the delivery of PK-L4 and investigate its biodistribution behavior after intravenous administration.

Results: The particle size of the PK-L4-loaded SLNs was $47.3 \mathrm{~nm}$ and the size of the PEGylated form was smaller, at $28 \mathrm{~nm}$. The entrapment efficiency (EE\%) of PK-L4 in SLNs with and without PEG showed a high capacity of approximately $100 \%$ encapsulation. Results also showed that the amount of PK-L4 released over a prolonged period from SLNs both with and without PEG was comparable to the non-formulated group, with $16.48 \%$ and $30.04 \%$, respectively, of the drug being released, which fit a zero-order equation. The half-maximal inhibitory concentration values of PK-L4-loaded SLNs with and those without PEG were significantly reduced by $45 \%-64 \%$ in the human lung carcinoma cell line (A549), 99\% in the human breast adenocarcinoma cell line with estrogen receptor (MCF7), and 95\% in the human breast adenocarcinoma cell line (MDA-MB-231). The amount of PK-L4 released by SLNs with PEG was significantly higher than that from the PK-L4 solution $(P<0.05)$. After intravenous bolus of the PK-L4-loaded SLNs with PEG, there was a marked significant difference in half-life alpha ( $0.136 \pm 0.046$ hours) when compared with the PK-L4 solution ( $0.078 \pm 0.023$ hours); also the area under the curve from zero to infinity did not change in plasma when compared to the PK-L4 solution. This demonstrated that PK-L4-loaded SLNs were rapidly distributed from central areas to tissues and exhibited higher accumulation in specific organs. The highest deposition of PK-L4-loaded SLNs with PEG was found in the lung and spine.

Conclusion: Sufficient amounts of PK-L4 were entrapped in the SLNs, and the pharmacokinetic behavior of PK-L4-loaded SLNs was established. This formulation successfully resolved the delivery problem, and the drug was localized in particular organs.

Keywords: 3-chloro-4-[(4-methoxyphenyl)amino]furo[2,3-b]quinoline, SLNs, solubility, cytotoxicity, biodistribution

\section{Introduction}

Amsacrine, a chemotherapeutic drug that is a substituted 9-aminoacridine derivative, is mainly used to treat refractory acute leukemia and lymphomas. ${ }^{1,2}$ However, as the half-life of amsacrine in plasma is around 20-30 minutes, clinical use is limited. ${ }^{3}$ 
Moreover, toxicity problems occur in the heart, and nausea, vomiting, and diarrhea are common side effects. Previous research has focused on synthesizing a series of structureactivity relationships (SARs) for amsacrine analogs, such as DNA-intercalated 9-anilinoacridine derivatives, to increase the potential antitumor activity, decrease side effects, and prolong the half-life. ${ }^{4,5}$

In a past study, Chen et al successfully synthesized a series of amsacrine analogs, among which 3-chloro-4-[(4methoxyphenyl)amono]furo[2,3-b]-quinoline (PK-L4) exhibited potential antitumor activity and also overcame certain disadvantages of amsacrine. ${ }^{6}$ In terms of the SARs, acridine is the backbone of amsacrine's structure and is related to its antitumor activity. Past evidence has demonstrated that the furo[2,3-b] quinoline ring and acridine are equivalence isomers. Since furo[2,3-b]quinoline derivatives have higher electron densities than acridine, they have longer half-lives. ${ }^{7}$ According to the results of cytotoxicity evaluations by the National Cancer Institute (NCI), PK-L4 acts against most cancers including non-small-cell lung cancer (NCI-H-460), central nervous system cancer (SF-268), and breast cancer (MCF-7). PK-L4 has powerful antitumor activity and the half-maximal inhibitory concentration $\left(\mathrm{IC}_{50}\right)$ is $0.025 \mu \mathrm{M} .^{8,9}$ Its mechanism is related to DNA intercalation, as it causes double-strand breaks in the DNA, and inhibits topoisomerase II, leading to arrest at the S and $\mathrm{G} 2$ phases of the cell cycle. ${ }^{10}$

In spite of its attractive antitumor activities, PK-L4 is water insoluble and does not dissolve in most biocompatible media, thus making it difficult to deliver to cancer cells. ${ }^{11}$ Generally, useful preliminary criteria for a suitable candidate from which to prepare solid lipid nanoparticles (SLNs) are that the compound should have low solubility and low permeability. ${ }^{12}$ Lipophilic drugs are potential candidates for SLNs as they gain access to the systemic circulation via a lymphatic route, which avoids hepatic first-pass metabolism. SLNs were introduced in the early 1990s and previous literature has demonstrated that they can improve a drug's bioavailability, especially of a compound with poor water solubility; prolong the systemic circulation time; control its release; and change its distribution properties. ${ }^{13}$ As is wellknown, one of the disadvantages of typical chemotherapeutic drugs is that they affect healthy cells, which creates temporary or longer-term side effects. An adequate distribution to specific sites in the body must be achieved, and effective drug concentrations must be maintained at those sites for the required period of time. ${ }^{14}$

Chen et al previously successfully synthesized PK-L4, confirmed its structure by nuclear magnetic resonance
(NMR), and demonstrated its actions against several cancer cell types. ${ }^{6}$ Our current objective was to prepare PK-L4loaded SLNs to improve solubility and investigate the pharmacokinetics and organ distribution after intravenous administration.

\section{Materials and methods Materials}

PK-L4 was synthesized in the laboratory of the School of Medicinal and Applied Chemistry (Kaohsiung Medical University, Kaohsiung, Taiwan). Pluronic ${ }^{\circledR}$ F 68 (F-68) and diclofenac were purchased from Sigma-Aldrich (St Louis, MO). Trimyristin (TM) was purchased from Tokyo Chemical Industry Co, Ltd (Tokyo, Japan). Hydrogenated soybean phosphatidylcholine (HSPC, 90\%) was purchased from Nippon Oil \& Fats Co., Ltd. (NOF) (Tokyo, Japan). Gelucire ${ }^{\circledR} 53 / 10$ was obtained from Gattefossé (Gennevilliers, France) and and 1-Pentanesulfonic acid sodium salt and polyethylene glycol (PEG)-monostearate E.O.(45) were obtained from Wako Pure Chemical Industries, Ltd., (Osaka, Japan). Methanol, acetonitrile, dichloromethane, and other chemicals were of analytical reagent grade.

A fibroblast cell line (Hs68) was purchased from the Culture Collection and Research Center of the Food Industry Research and Development Institute (Hsinchu, Taiwan). Cell culture media and supplements were obtained from GIBCO Invitrogen (Carlsbad, CA).

\section{Methods}

\section{Preparation of SLNs}

SLNs were prepared by hot homogenization followed by an ultrasonication method. The oil phase consisted of TM, Gelucire 53/10, HSPC, stearylamine, and PEG monostearate, and the model drug (PK-L4) was pre-dissolved in $3 \mathrm{~mL}$ of a 1:1 mixture of chloroform and methanol in order to ensure it was well mixed. Organic solvents were completely removed under nitrogen for 30 minutes. The aqueous phase with double-distilled water contained 1\% F-68. The two phases were heated separately to $80^{\circ} \mathrm{C}$. The aqueous phase was added to the lipid phase and mixed using a probetype sonicator (UP50H, Hielscher Ultrasonics, Teltow, Germany) for 20 minutes at $50 \mathrm{~W}$. The resulting suspensions were cooled down at room temperature $\left(25^{\circ} \mathrm{C}\right)$ then filtered through a membrane with a $0.22 \mu \mathrm{m}$ pore size $(25 \mathrm{~mm}$ filter, Phenomenex, Torrance, CA). The compositions of the SLNs are given in Table 1. The PK-L4 control solution used $300 \mu \mathrm{L}$ ethanol, $900 \mu \mathrm{L}$ PEG 400, $1200 \mu \mathrm{L}$ tetraglycol, and 
Table I Composition of the sold lipid nanoparticles (SLNs) grafting with or without polyethylene glycol (PEG)

\begin{tabular}{|c|c|c|c|c|c|c|c|}
\hline \multirow[t]{2}{*}{ Formulations } & \multicolumn{6}{|c|}{ Lipid phase (mg, \%) } & \multirow{2}{*}{$\begin{array}{l}\text { Aqueous phase }(\mu \mathrm{L}) \\
\% \text { F-68 }\end{array}$} \\
\hline & Trimyristin & $\begin{array}{l}\text { Gelucire }^{\circledR} \\
53 / 10\end{array}$ & $\begin{array}{l}\text { Hydrogenated } \\
\text { SPC }\end{array}$ & Stearylamine & $\begin{array}{l}\text { PEG } \\
\text { monostearate }\end{array}$ & PK-L4 & \\
\hline SLNs without PEG & $60(2 \%)$ & $60(2 \%)$ & $30(1 \%)$ & $1.5(0.05 \%)$ & - & $4.5(0.15 \%)$ & 2820 \\
\hline SLNs with PEG & $60(2 \%)$ & $60(2 \%)$ & $30(1 \%)$ & $1.5(0.05 \%)$ & $30(1 \%)$ & $4.5(0.15 \%)$ & 2820 \\
\hline
\end{tabular}

Abbreviations: F-68, Pluronic ${ }^{\circledR}$ F 68; PK-L4, 3-Chloro-4-[(4-methoxyphenyl)amino]furo[2,3-b]quinoline; SPC, soybean phosphatidylcholine.

$600 \mu \mathrm{L}$ double-distilled water as the medium to dissolve $9 \mathrm{mg}$ of PK-L4.

\section{Particle size and zeta potential}

The mean diameter $(d)$ and zeta potential of the SLNs were measured by photon correlation spectroscopy (Zetasizer 3000HS, Malvern Instruments, Malvern, UK) using a helium-neon laser with a wavelength of $633 \mathrm{~nm}$ at $25^{\circ} \mathrm{C}$. The size values are given as a volume distribution. All vesicles were diluted 200-fold with double-distilled water for the measurements. The determination was repeated three times per sample for three samples.

\section{$\mathrm{EE} \%$}

The EE\% of PKL4 in SLNs was determined by an ultracentrifugation method. The systems were centrifuged at 120,000 rpm for $2 \mathrm{~h}$ at $4^{\circ} \mathrm{C}$ in a Hitachi CS150 GXL ultracentrifuge (Tokyo, Japan) to separate the incorporated drug from the free form. The supernatant was analyzed by high-performance liquid chromatography (HPLC) to determine the EE\%. Drug $\mathrm{EE} \%$ were calculated by the equation:

$$
\begin{aligned}
\text { E.E. } \%= & ((\text { Total amount-Free amout }) /(\text { Total amount })) \\
& \times 100 \%
\end{aligned}
$$

\section{Storage stability}

The prepared SLN colloids were stored at room temperature $\left(25^{\circ} \mathrm{C}\right)$ for 1 month. An aliquot of a sample was taken at predetermined time intervals to investigate the particle size, polydispersity index (PDI), zeta potential, and entrapment percentage. The mean size was determined by photon correlation spectroscopy as described in the previous section. The concentration of PK-L4 was determined by ultracentrifugation to understand changes in the drug entrapment percentage.

\section{Transmission electron microscopy (TEM)}

The morphology of the SLNs was observed by TEM to characterize the microstructure. An SLN suspension $(200 \mu \mathrm{L})$ was added to a carbon film-covered copper grid (Ted Pella Inc, Redding, CA), which was stained with $1 \%$ phosphotungstic acid for 20 minutes, washed with double-distilled water, and placed in a vacuum overnight. The sample was then examined using a JEOL JEO-2000EX II transmission electron microscope (Tokyo, Japan).

\section{Differential scanning calorimetry (DSC)}

In DSC, the broad water peak $\left(100^{\circ} \mathrm{C}\right)$ largely influences the interpretation of thermograms of lipid nanoparticles. To avoid this interference, the SLNs were freeze-dried FreeZone $^{\circledR}$ (Labconco, Kansas, MO, USA) before the DSC measurements. The DSC analysis was performed using a Q10 differential scanning calorimeter. All formulation ingredients (5 mg) and freeze-dried SLNs (2 mg) were put into aluminum pans, and the temperature was increased from $30^{\circ} \mathrm{C}$ to $80^{\circ} \mathrm{C}$ or $30^{\circ} \mathrm{C}$ to $180^{\circ} \mathrm{C}$ at a rate of $10^{\circ} \mathrm{C} / \mathrm{min}$ under nitrogen purge. An empty pan was used as a reference.

\section{PK-L4 analysis by HPLC}

Apparatus and chromatographic conditions

HPLC was carried out using a Hitachi model L-700 pump, a BAS LC-4C electrochemical detector (ECD) (BAS, Tokyo, Japan), a JASCO 855-AS auto-sampler (Tokyo, Japan), and a Lichrocart ${ }^{\circledR} \mathrm{C} 18 \mathrm{e}$ column $(55 \mathrm{~mm} \times 4 \mathrm{~mm}$ internal diameters (ID), $3 \mu \mathrm{m}$ ) (Merck, Darmstadt, Germany). The mobile phase was a mixture of $45 \%$ acetonitrile and $55 \%$ of a $0.01 \mathrm{M}$ 1-pentansulfonic acid sodium salt solution (v/v, $\mathrm{pH} 3.0)$, and the flow rate was $1.0 \mathrm{~mL} / \mathrm{min}$. The effluent from the column was monitored by an electron capture detector at $1.0 \mathrm{~V}$ and 10 nA. A BAS MF 1000 dual glassy carbon working electrode and DAS RE6 Ag/AL reference electrode were used ECD system (West Lafayette, IN, USA). Diclofenac prepared at a concentration of $0.5 \mu \mathrm{g} / \mathrm{mL}$ was the internal standard (IS).

\section{Liquid chromatographic (LC) method validation}

The limit of detection (LOD) and limit of quantitation (LOQ) of PK-L4 were determined by dissolving it at decreasing concentrations in acetonitrile until the signal-to-noise $(\mathrm{S} / \mathrm{N})$ ratios were 3 and 10, respectively. The recovery of PK-L4 from plasma was assessed by comparing the peak area obtained from spiked PK-L4 samples with those standards in a dichloromethane solution that was processed in the 
same way as the samples. Standard samples of rat plasma were prepared and analyzed during method validation. The linearity of the standard curves, and the intra-day and inter-day precision and accuracy were determined from six concentrations of PK-L4 of $0.1,0.3,0.5,1,3$, and $5 \mu \mathrm{g} / \mathrm{mL}$ under the conditions previously described.

\section{In vitro release study}

Cellulose dialysis membranes with a molecular weight cutoff of 12,000-14,000 Daltons (Orange Scientific, Houston, TX) were soaked in double-distilled water for 12 hours before the experiment. PK-L4 SLN suspensions $(1 \mathrm{~mL})$ were put into dialysis bags and subsequently placed in flasks containing $100 \mathrm{~mL}$ medium ( $\mathrm{pH} 7.4$ phosphate buffer containing 1\% Pluronic F 127 [F-127]) to maintain sink conditions during the experiments in a $37^{\circ} \mathrm{C}$ water bath. At appropriate intervals, $1 \mathrm{~mL}$ aliquots of the medium were withdrawn from the receiver compartment through a side tube and immediately replaced with an equal volume of fresh medium. The amounts of the drug released were analyzed by the HPLC method described.

\section{$I_{50}$ values as determined by a cytotoxicity assay of PK-L4 and encapsulated SLNs}

We employed PK-L4 with and without SLNs in five different cell lines to confirm its potential for cellular cytotoxicity. The human lung carcinoma (A549), human hepatocellular liver carcinoma (HepG2), human breast adenocarcinoma with estrogen receptor (MCF7), human breast adenocarcinoma (MDA-MB-231), and human oral squamous cell carcinoma (Ca9-22) cell lines were maintained in Dulbecco's modified Eagle's medium (DMEM) with 10\% fetal bovine serum (FBS) and $1 \%$ antibiotics in a humidified atmosphere containing $5 \%$ $\mathrm{CO}_{2} / 95 \%$ air at $37^{\circ} \mathrm{C}$. Cells were seeded on 96 -well plates at a cell density of $5 \times 10^{4}$ cells/well, except for A549, which was seeded at $5 \times 10^{3}$ cells/well until cells became confluent. Fifty microliters of PK-L4 with and without the SLN suspensions at a concentration of $40 \mu \mathrm{g} / \mathrm{mL}$ were added and incubated for 72 hours. After washing with phosphate-buffered saline (PBS), $100 \mu \mathrm{L}$ of an 3-(4,5-Dimethylthiazol-2-yl)-2,5diphenyltetrazolium bromide (MTT) solution $(25 \mu \mathrm{g} / \mathrm{mL})$ was pipetted into each well, the plates were incubated for 2 hours, and then $100 \mu \mathrm{L}$ dimethyl sulfoxide was added to lyse the formazan crystals. The quantity of surviving cells was measured by an enzyme-linked immunosorbent assay reader at $550 \mathrm{~nm}$. The $\mathrm{IC}_{50}$, the drug concentration at which $50 \%$ growth inhibition is achieved, was calculated using a four-parameter logistic function standard curve analysis of the dose response.

\section{Animals and drug administration}

Male Wistar rats (obtained from the Laboratory Animal Center of the National Science Council, Taipei, Taiwan) weighing 200-250 g were used in the study. The animal experiment protocol was reviewed and approved by the Institutional Animal Care and Use Committee of Kaohsiung Medical University. Animals were housed and handled according to institutional guidelines. All animals were fasted overnight prior to the experiments. Rats were anesthetized with $25 \%$ urethane $(3 \mathrm{~mL} / \mathrm{kg})$ before the experiment. PK-L4 with and without SLNs was administered via an injection to the jugular vein at a dose of $4.7 \mathrm{mg} / \mathrm{kg}$. Rats were sacrificed after administration of PK-L4 to the control group or PK-L4loaded SLNs to the experimental group.

\section{In vivo pharmacokinetics}

Blood samples $(0.3 \mathrm{~mL})$ were collected from the heart at specified time intervals after PK-L4 administration with and without SLNs. Blood samples were then centrifuged at $3000 \mathrm{rpm}$ for 10 minutes at $4^{\circ} \mathrm{C}$, and plasma was immediately separated and stored at $-20^{\circ} \mathrm{C}$ until analysis. Aliquots of blank and plasma samples at $100 \mu \mathrm{L}$ were mixed with $50 \mu \mathrm{L}$ of $5 \mu \mathrm{g} / \mathrm{mL}$ of an IS solution and $4 \mathrm{~mL}$ dichloromethane as the extraction solvent. Rats were sacrificed after administration of PK-L4 with and without SLNs at specified time intervals. Tissues and organs including the heart, lungs, liver, spleen, kidneys, whole brain, spine, and small and large intestines were harvested and weighed on an analytical balance. The drug in the tissues and organs was extracted by a homogenization method. Normal saline $(1.5 \mathrm{~mL})$ was added to the excised tissue in a homogenizer at $26,000 \mathrm{rpm}$ for 30 seconds then $50 \mu \mathrm{L}$ of $5 \mu \mathrm{g} / \mathrm{mL}$ IS and $6 \mathrm{~mL}$ dichloromethane were added for extraction. The mixture was horizontally shaken at $200 \mathrm{rpm}$ for 1 hour and centrifuged at $3000 \mathrm{rpm}$ for 10 minutes. A total of $2.5 \mathrm{~mL}$ of the organic layer was transferred to another tube and evaporated to dryness under a vacuum. The dry residue was reconstituted in $150 \mu \mathrm{L}$ acetonitrile, and $20 \mu \mathrm{L}$ of clear supernatant was injected into the HPLC system.

\section{Analysis of pharmacokinetic data}

The pharmacokinetics were calculated on each individual set of data, using the pharmacokinetic software WinNonLin (v 4.0; Pharsight Software, Mountain View, CA) to determine the type of compartment model using the correlation coefficient from the observed and predicted data. The pharmacokinetic data measured included the area under the plasma concentration-time curves from time zero to infinity $\left(\mathrm{AUC}_{0-\infty}\right)$, the half-life of the distribution phase $\left(\mathrm{t}_{1 / 2 \alpha}\right)$, the 
half-life of the elimination phase $\left(\mathrm{t}_{1 / 2 \beta}\right)$, and the clearance $(\mathrm{Cl})$. The $\mathrm{AUC}_{0-\infty}$ was calculated using the trapezoidal rule $\left(\mathrm{AUC}_{0-\mathrm{t}}\right)$ and was extrapolated to infinity by the addition of $\mathrm{AUC}_{\mathrm{t}-\infty}$. The $\mathrm{k}$ value was the terminal slope, which was calculated by a linear regression of the logarithmic value of the terminal phase. The $\mathrm{Cl}$ value was calculated as the dose/ $\mathrm{AUC}_{0-\infty}$, and the mean residence time (MRT) and the volume of distribution at steady state (Vss) were obtained by summation of the central and tissue compartments.

\section{Statistical analysis}

All data reported are expressed as the mean \pm standard derivation. Statistical analyses were performed using an unpaired Student's $t$-test with Winks SDA 6.0 software (Texasoft, Duncanville, TX). An analysis of variance test was also used. Subgroup comparisons were made using the Newman-Keuls multiple comparisons. A 0.05 level of probability was used as the level of significance.

\section{Results}

\section{Physicochemical characterization of SLNs}

We evaluated the mean diameter, PDI, zeta potential, and EE of PK-L4-loaded SLNs. Table 1 summarizes the composition of the SLNs with and SLNs without PEG. The physicochemical properties are shown in Table 2. The particle size of SLNs was $47.3 \mathrm{~nm}$, and when incorporated with PEG, this was reduced to $28 \mathrm{~nm}$. The PDI showed a narrow distribution in the range of $0.13-0.18$. The zeta potential of SLNs and those incorporating PEG presented positive charges at 22.5 and $27.8 \mathrm{mV}$, respectively. The EE\% of SLNs with and SLNs without PEG showed a high capacity for carrying PK-L4 of approximately $100 \%$. To obtain more information about the particle size and morphology, TEM analysis was also performed. Figure 1A and B show the TEM morphology of SLNs with PEG in microscopic view. The images reveal that the SLNs had nanometer-sized spherical outlines, uniform shapes, and a well-dispersed pattern. Figure $1 \mathrm{C}$ presents a schematic depiction of the PK-L4-loaded SLN system.

\section{Storage stability}

The physical stability of SLNs was determined by measuring particle sizes at 0 and 1 month, as shown in Table 2. Results showed that particle sizes of SLNs with and SLNs without PEG remained relatively stable after 1 month of storage $(P>0.05)$ as did the zeta potential. However, the PDI of the SLN without PEG group. slightly increased compared with the PEG group. The EE\% of the SLNs with and SLNs without PEG after storage decreased about by $19.1 \%$ and $8.35 \%$, respectively. Nevertheless, SLNs both with and without PEG generally maintained drug capacity at about $80 \%-90 \%$.

\section{DSC analysis}

The thermal behavior of the conjugates was investigated using DSC as shown in Figure 2. The DSC curves of TM, Gelucire 53/10, stearylamine, and PEG monostearate showed endothermic peaks at $50^{\circ} \mathrm{C}-80^{\circ} \mathrm{C}$, which were assigned to their melting points. HSPC did not present a significant peak in that temperature range. PK-L4 in its natural state presented a high peak at its melting point $\left(180^{\circ} \mathrm{C}\right)$. However, when entrapped in SLNs with and without PEG, the peak at the original melting point disappeared. These results demonstrate that PK-L4 was covalently conjugated to SLNs incorporating PEG. ${ }^{15}$

\section{In vitro release kinetics}

Release kinetics of PK-L4 from the SLN carrier were determined as the release percentage, and the best-fitting kinetics model was calculated to explore the release behavior, as shown in Figure 3. Three kinds of receptor medium were selected to confirm the solubility of PK-L4: pH 7.4 PBS, $\mathrm{pH}$ 7.4 PBS containing 1\% F-68, and $\mathrm{pH}$ 7.4 PBS containing $1 \% \mathrm{~F}-127$. Results showed the $\mathrm{pH} 7.4$ PBS containing 1\% F-127 exhibited the best solubility; hence, we chose it as the receptor medium. The release data showed that the PK-L4 solution fitted the Higuchi order. Moreover, the result also showed that prolonged PK-L4 release from SLNs with and those without PEG compared with the non-formulated

Table 2 Characterization of 3-Chloro-4-[(4-methoxyphenyl)amino]furo[2,3-b]quinoline-loaded solid lipid nanoparticles (SLNs) with or without polyethylene glycol (PEG) in terms of the diameter of particle size (d), polydispersity index (PDI), zeta potential, and entrapment efficiency $(\mathrm{EE} \%)$, and stability changes following storage at room temperature $\left(25^{\circ} \mathrm{C}\right)$ for I month

\begin{tabular}{|c|c|c|c|c|c|c|c|c|}
\hline \multirow[t]{2}{*}{ Formulation } & \multicolumn{2}{|l|}{$d(\mathrm{~nm})$} & \multicolumn{2}{|l|}{ PDI } & \multicolumn{2}{|c|}{ Zeta potential $(\mathrm{mV})$} & \multicolumn{2}{|l|}{ EE\% } \\
\hline & 0 day & I month & 0 day & I month & 0 day & I month & 0 day & I month \\
\hline SLNs without PEG & $47.3 \pm 0.5$ & $42.4 \pm 0.4$ & $0.18 \pm 0.01$ & $0.18 \pm 0.01$ & $22.5 \pm 1.5$ & $20.9 \pm 1.8$ & $99.69 \pm 0.12$ & $80.59 \pm 1.20$ \\
\hline SLNs with PEG & $28.0 \pm 0.6$ & $28.7 \pm 0.3$ & $0.13 \pm 0.02$ & $0.20 \pm 0.01$ & $27.8 \pm 6.4$ & $25.4 \pm 3.3$ & $99.98 \pm 0.00$ & $91.63 \pm 2.36$ \\
\hline
\end{tabular}

Note: Each value represents the mean \pm standard deviation $(n=3)$. 
A

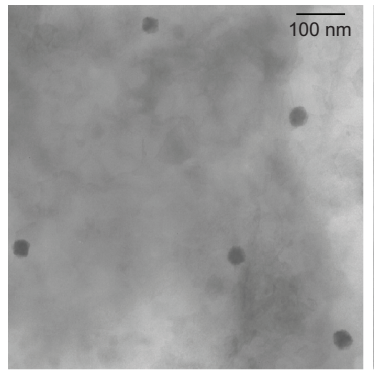

B

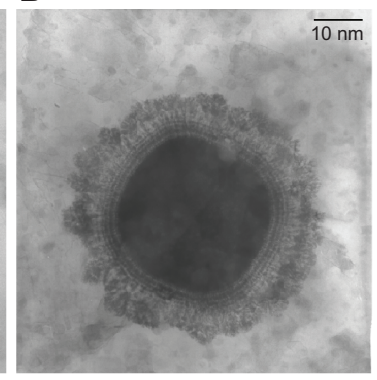

C

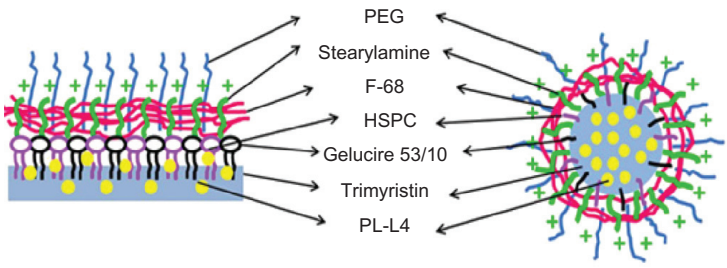

Figure I (A and B) Transmission electron microscopy images of 3-Chloro-4-[(4methoxyphenyl)amino]furo[2,3-b]quinoline (PK-L4)-loaded solid lipid nanoparticles (SLNs) with polyethylene glycol (PEG)-monostearate: (A) macro view, original magnification (50,000X); (B) microscopic view (70,000X). (C) Schematic depiction of a PK-L4-loaded SLN system.

Notes: The PK-L4 (yellow particle) entrapped in the inner cores of the SLNs (light blue) with Gelucire ${ }^{\circledR}$ 53/I0 (black), hydrogenated soybean phosphatidylcholine (HSPC) (purple), and the positive charge of stearylamine chelated in the core-shell, Pluronic ${ }^{\circledR}$ F 68 (F-68) (pink), connected to those of the surfactant, and functionalized with PEG (blue) (left), followed by the assembly of those of the surfactant on the core shells of the SLNs (right). The fully assembled PK-L4-loaded SLN system is shown on the right.

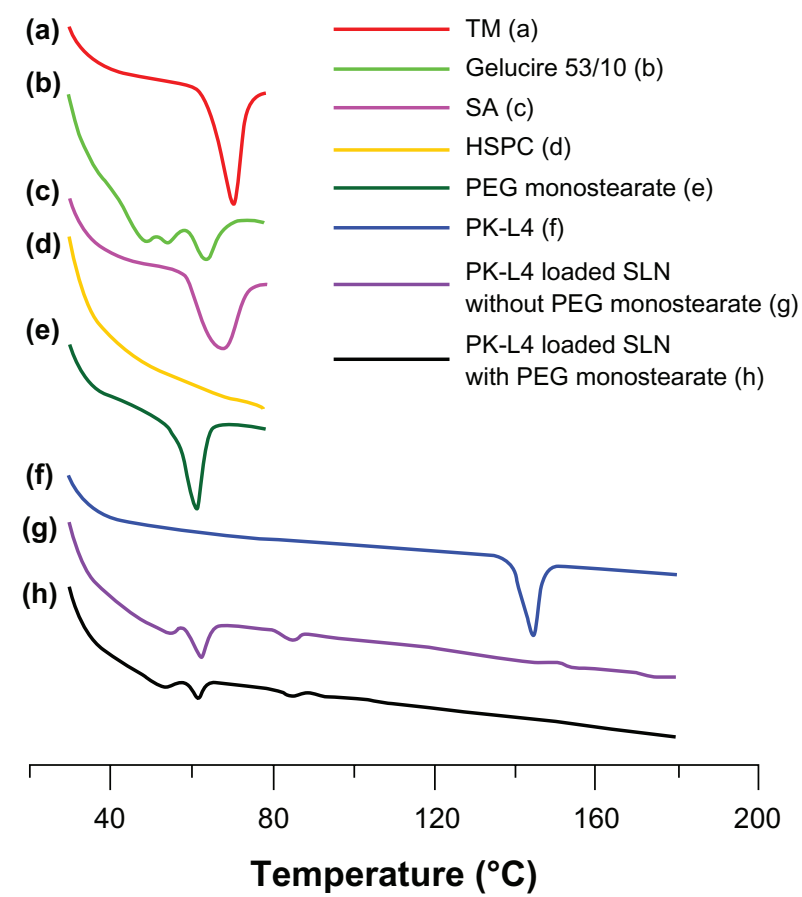

Figure 2 Differential scanning calorimetry thermograms of solid lipid nanoparticle (SLN) ingredients and 3-Chloro-4-[(4-methoxyphenyl)amino]furo[2,3-b]quinoline (PK-L4) at increasing scanning periods at $80^{\circ} \mathrm{C}-180^{\circ} \mathrm{C}$.

Abbreviations: HSPC, hydrogenated soybean phosphatidylcholine; PEG, polyethylene glycol; SA, stearylamine; TM, trimyristin.

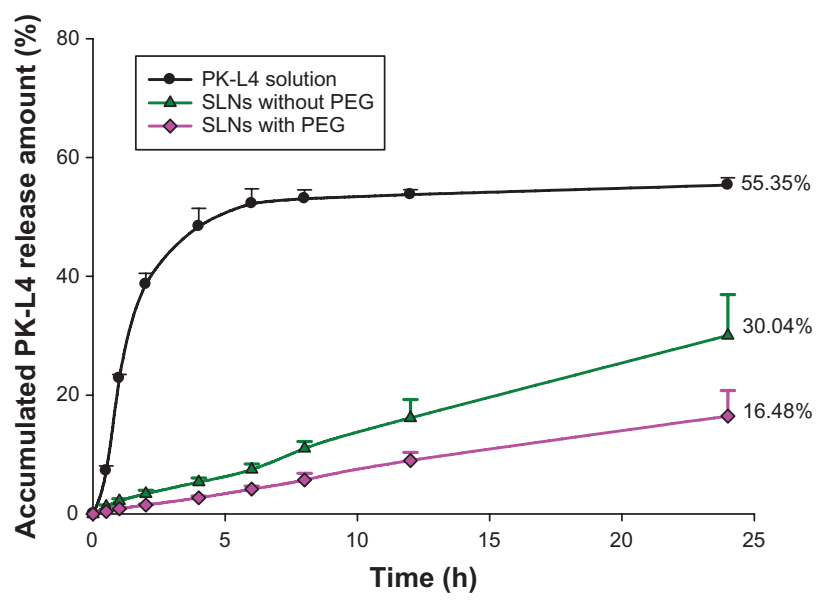

Figure 3 In vitro release of 3-Chloro-4-[(4-methoxyphenyl)amino]furo[2,3-b] quinoline (PK-L4) from solid lipid nanoparticles (SLNs) with and without polyethylene glycol (PEG) monostearate.

Notes: Phosphate-buffered saline ( $\mathrm{pH} 7.4)$ containing I\% Pluronic ${ }^{\circledR}$ F I 27 was used as the receptor medium. Each value represents the mean \pm standard deviation $(n=4)$.

group, and $16.48 \%$ and $30.04 \%$, respectively, of the drug was released over a period of 24 hours. Calculation of the release period fitted a zero-order equation, and the respective relative coefficients of SLNs with and without PEG were 0.9401 and 0.9312 , respectively. There was a significant difference between SLNs with and those without PEG monostearate, and non-formulated PK-L4 $(P<0.05)$.

\section{Cytotoxicity assay}

The in vitro cytotoxic activities of the PK-L4 solution and PK-L4-loaded SLNs with and without PEG were examined as shown in Table 3. The study screened five cell lines: A549, Hep G2, MCF7, MDA-MB-231, and Ca9-22. We also used a commercial potential anticancer drug as a positive control group. The $\mathrm{IC}_{50}$ of the PK-L4 solution was significantly lower than that of the doxorubicin solution in the A549, Hep G2, and Ca9-22 cell lines, and the $\mathrm{IC}_{50}$ values decreased to $73 \%$, $64 \%$, and $72 \%$, respectively $(P<0.05)$. Compared with the PK-L4 solution, $\mathrm{IC}_{50}$ values for PK-L4-loaded SLNs with and without PEG decreased to $45 \%-64 \%$ in the A549 line $(P<0.05)$, to $99 \%$ in the MCF7 line $(P<0.01)$, and $95 \%$ in the MDA-MB-231 line $(P<0.01)$ - that is, significant reductions in $\mathrm{IC}_{50}$ values were shown.

\section{Pharmacokinetics}

The LOD and LOQ for quantifying PK-L4 were 1 and $5 \mathrm{ng} / \mathrm{mL}$, respectively. The linear range was $0.02-3 \mu \mathrm{g} / \mathrm{mL}$ $\left(R^{2}=0.9986\right)$ in plasma. The pharmacokinetic parameters of the PK-L4 solution and PK-L4-loaded SLNs with and without 
Table 3 The half-maximal inhibitory concentrations $\left(\mathrm{IC}_{50}\right)$ of the 3-Chloro-4-[(4-methoxyphenyl)amino]furo[2,3-b]quinoline (PK-L4) solution and PK-L4-loaded solid lipid nanoparticles (SLNs) with and without polyethylene glycol (PEG) monostearate

\begin{tabular}{llllll}
\hline Formulations & \multicolumn{1}{l}{$\mathbf{I C}_{50}(\mu \mathrm{g} / \mathrm{mL})$ on cell lines } & & \\
\cline { 2 - 6 } & A549 & HepG2 & MCF7 & MDA-MB-23I & Ca9-22 \\
\hline PK-L4 solution & 0.11 & 0.04 & 8.47 & 2.14 & 0.04 \\
SLNs without PEG & 0.06 & 0.09 & $0.10^{* *}$ & $0.1 I^{* *}$ & 0.03 \\
SLNs with PEG & $0.04^{*}$ & 0.04 & $0.10^{* *}$ & $0.12^{* *}$ & 0.03 \\
Doxorubicin solution & $0.40^{*}$ & $0.11^{*}$ & $0.43^{* *}$ & $0.5 I^{* *}$ & $0.14^{*}$ \\
\hline
\end{tabular}

Notes: A doxorubicin solution was used as the comparison group. Each value represents the mean \pm standard deviation $(\mathrm{n}=6)$. $* P<0.05$; $* * P<0.0 \mathrm{I}$ as compared with the data of PK-L4 control solution. Note that doxorubicin solution was a free aqueous solution - this drug has commercial potential as an anticancer drug.

Abbreviations: A549, human lung carcinoma cell line; HepG2, human hepatocellular liver carcinoma cell line; MCF7, human breast adenocarcinoma with estrogen receptor cell line; MDA-MB-23I, human breast adenocarcinoma cell line; Ca9-22, human oral squamous cell carcinoma cell line.

PEG after an intravenous bolus are summarized in Table 4. The pharmacokinetics of PK-L4 best fit a two-compartment model based on the lower Akaike information criterion and higher $R^{2}$ values $(r=0.9920)$. After an intravenous bolus of the PK-L4 solution and SLNs with and without PEG, there was no significant difference in the $\mathrm{AUC}_{0-\infty}$ values in plasma. However, SLNs with and without PEG displayed a marked difference in the distribution phase $\left(\mathrm{t}_{1 / 2 \alpha}=0.136 \pm 0.046\right.$ and $0.118 \pm 0.009$ hours, respectively) compared with the PK-L4 solution $\left(t_{1 / 2 \alpha}=0.078 \pm 0.023\right.$ hours $)$. Moreover, the elimination phases $\left(t_{1 / 2 \beta}\right)$ of SLNs with and without PEG monostearate were also slightly prolonged compared with the PK-L4 solution $(P>0.05)$. Both the plasma Vss and MRT of SLNs with and without PEG monostearate were significantly lower than those of the PK-L4 solution $(P<0.05)$.

\section{Biodistribution}

After intravenous administration of the PK-L4 solution and PK-L4-loaded SLNs with and without PEG, tissues and organs were homogenized to extract PK-L4, and the linear range was $0.03-10.0 \mu \mathrm{g} / \mathrm{g}\left(R^{2}=0.9949-0.9989\right)$. As shown in Figure 4, the PK-L4 solution was mainly located in the spine, followed by the lungs, brain, small intestine, and heart. Moreover, the PK-L4 concentration in SLNs with PEG was significantly higher than that of the PK-L4 solution in the heart, lungs, and large intestine $(P<0.05)$. The highest deposition of PK-L4-loaded SLNs with PEG group was found in the lungs and spine.

\section{Discussion}

Amsacrine, a chemotherapeutic agent, promotes remission of acute leukemia in the clinic. Unfortunately, a low therapeutic index, a short half-life, and toxicity after metabolism limit its clinical applications. Previous studies have demonstrated PK-L4 to be a potential anticancer drug active against several solid and liquid tumors, with a longer halflife than amsacrine. ${ }^{8,9}$ However, a powerful $\mathrm{IC}_{50}$ produces a narrow therapeutic index, and PK-L4 is water insoluble in most biocompatible media used for intravenous injection, which obstructs its delivery into cancer cells in the biocirculation. Hence, the selection of a suitable carrier and design of its formulation are necessary to overcome some of these disadvantages. In this study, sufficient amounts of PK-L4 were entrapped in the SLNs, and the pharmacokinetic behavior of PK-L4-loaded SLNs was established. This formulation successfully resolved the delivery problem, and the drug was localized in particular organs.

Table 4 Pharmacokinetic parameters of 3-Chloro-4-[(4-methoxyphenyl)amino]furo[2,3-b]quinoline (PK-L4) (4.7 mg/kg) after intravenous administration of the control (PK-L4 solution) and solid lipid nanoparticles (SLNs) with and without polyethylene glycol (PEG) to rats for 12 hours

\begin{tabular}{|c|c|c|c|}
\hline Parameters & Control (PK-L4 solution) & SLNs without PEG & SLNs with PEG \\
\hline $\mathrm{t}_{1 / 2 \alpha}(\mathrm{h})$ & $0.078 \pm 0.023$ & $0.118 \pm 0.009^{*}$ & $0.136 \pm 0.046^{*}$ \\
\hline $\mathrm{t}_{1 / 2 \beta}(\mathrm{h})$ & $0.994 \pm 0.138$ & $1.042 \pm 0.118$ & $1.026 \pm 0.658$ \\
\hline $\mathrm{Cl}(\mathrm{L} / \mathrm{h} / \mathrm{kg})$ & $4.075 \pm 0.323$ & $4.148 \pm 0.265$ & $4.296 \pm 0.134$ \\
\hline Vss (L/kg) & $4.118 \pm 0.395$ & $4.457 \pm 0.065$ & $2.878 \pm 0.970^{*}$ \\
\hline $\mathrm{AUC}_{0 \rightarrow \infty}(\mathrm{h} \cdot \mu \mathrm{g} / \mathrm{mL})$ & $1.158 \pm 0.087$ & $1.136 \pm 0.07 \mid$ & $1.095 \pm 0.034$ \\
\hline MRT (h) & $1.016 \pm 0.145$ & $1.078 \pm 0.081$ & $0.674 \pm 0.244 *$ \\
\hline
\end{tabular}

Note: Each value represents the mean \pm standard deviation $(n=3)$.

Abbreviations: $\mathrm{AUC}_{0-\infty}$, area under the plasma concentration-time curve from time zero to infinity; $\mathrm{Cl}$, clearance; MRT, mean residence time; $\mathrm{t}_{1 / 2 \alpha}$, half-life of the distribution phase; $t_{1 / 2 \beta}$, half-life of the elimination phase; Vss, volume of distribution at steady state. 


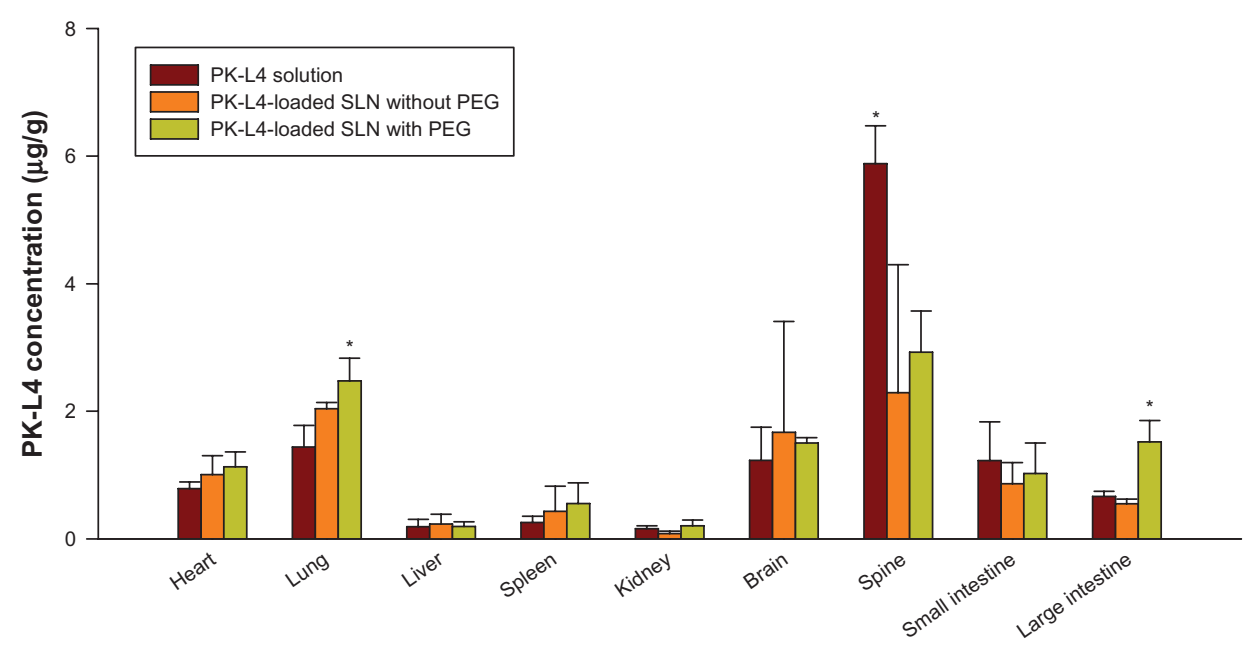

Figure 4 Tissue distribution of 3-Chloro-4-[(4-methoxyphenyl)amino]furo[2,3-b]quinoline (PK-L4) concentrations from a control PK-L4 solution and one loaded into solid lipid nanoparticles (SLNs) with or without polyethylene glycol (PEG) after intravenous administration to Wistar rats at a dose of $4.7 \mathrm{mg} / \mathrm{kg}$ for $30 \mathrm{minutes}$. Notes: Each value represents the mean and SD $(n=3)$. ${ }^{*}<<0.05$ as compared with the data of the PK-L4 control solution.

In terms of therapeutic outcomes, SLNs can be utilized to improve the therapeutic efficacy of drugs with low bioavailability, and reduce their effective dose requirements. Moreover, in terms of the preparation technique, SLN systems were introduced to overcome limitations associated with traditional emulsion formulations. Generally, pharmaceutically acceptable intravenously administered droplets are limited to $5 \mu \mathrm{m}$ in diameter. ${ }^{16} \mathrm{In}$ the strictest sense, "nanotechnology" refers to structures roughly in the $1-100 \mathrm{~nm}$ size range. ${ }^{17}$ In the present study, all of the SLN formulations exhibited diameters of $<50 \mathrm{~nm}$, and particles were well dispersed, which meant that the particle size could increase uptake by organs and avoid unpredictable toxicity from a broader particle-size distribution.

In the present study, the SLN formulations were composed of TM, Gelucire 53/10, HSPC, stearylamine, and F-68 with or without PEG monostearate. TM plays a role as the main digestible lipid in triglyceride lipids and is preferably hydrolyzed by pancreatic lipase, an interfacial enzyme, and gastric lipase; thus, it is partially relevant to the circulation time in the body. ${ }^{18}$ Gelucire 53/10 is PEG-32 glyceryl stearate. PEG glyceryl stearates are a family of lipid-based excipients comprising glycerides and esters of PEG, which confer hydrophobic and hydrophilic properties, respectively, to the vehicle. The hydrophilic-lipophilic balance value of Gelucire 53/10 was 10; therefore, when used in pharmaceutical excipients as a surfactant and lipid-based component, Gelucire 53/10 also provides sustained-release properties. HSPC and F-68 acted as emulsifiers in this formulation. SLNs with PEG produced a relatively smaller particle size than those without the PEG group. PEG monostearate has a
PEGylated effect, and contains a stearate group and a PEG spacer. The ability of amphiphilic PEG to act as a hydrophilic shield in drug carriers reduces the surface tension of particles, also reducing their sizes. ${ }^{19} \mathrm{~A}$ previous study investigated the relationship between the molecular weight of PEG and particle size, pointing out that as the molecular weight of PEG increased, particle size would decrease.$^{20}$ The incorporation of stearylamine into SLNs shielded the positive charge. To maintain the stability of the nanoparticles, a surface charge modifier should be incorporated. ${ }^{21}$ Another study mentioned that the zeta potential decreased from -15 to $-25 \mathrm{mV}$, which produced a gelation effect. ${ }^{22}$ In terms of our stability result, it indicated that after 1 month, the particle size and PDI remained in the original ranges.

TEM morphology of SLNs showed that the nanoparticles consisted of a hydrophilic outer shell and a hydrophobic inner core in aqueous media. The morphology also exhibited multiple layers surrounding the inner core, which was coated with F-68. These core-shell structures carried lipophilic drugs into the inner core and also provided sustained-release properties at a later stage, while the hydrophilic shell provided stability for the nanoparticles without the need for additional stabilizers, thus rendering them a potential carrier for drugs with poor water solubility. ${ }^{23}$ The PEG monostearate molecule is an amphiphilic polymeric derivative of hydrophilic PEG modified by attaching a hydrophobic moiety, which was easily incorporated into the lipid core of SLNs with hydrophilic PEG chains on their surface. ${ }^{24}$ Past research has documented that the circulation of nanoparticles can be prolonged by coating their surfaces with a hydrophilic polymer such as modified varieties of the molecular PEG series or F-68..$^{25,26}$ 
The schematic depiction of PK-L4-loaded SLN system are shown in Figure 1C.

The physical stability of the SLNs indicated that particle sizes and zeta potentials did not significantly differ, and preliminary results indicated that SLNs with and without PEG were stable in terms of particle size and charge shape. Moreover, measurement of the zeta potential allows the storage stability of colloidal dispersions to be predicted. A high zeta potential of $>30 \mathrm{mV}$ can provide electric repulsion that helps prevent particle aggregation. ${ }^{27,28}$ Hence, one possible reason for the stability may have been the high zeta potential. Results showed that PK-L4 had a high $\mathrm{EE} \%$ of about $100 \%$ in the SLNs; however, after 1 month of storage, the EE\% had decreased by about ten- to 20 -fold, which we assumed was due to the solubilization of the lipid crystallization.

DSC was used to understand the interactions between PK-L4 and the SLN formulations. Thermograms of PK-L4 were about $140^{\circ} \mathrm{C}$, and PK-L4-loaded SLNs with and without PEG monostearate did not show a melting peak for PK-L4 of around $140^{\circ} \mathrm{C}$. This suggests that PK-L4 was in an amorphous or molecular state rather than a crystalline state - that is, that the PK-L4 was homogeneously dispersed in the core lipid. Consequently, the type of surfactant and storage time affected the crystallinity of SLNs and the degradation velocity. ${ }^{29}$ Polymorphic transitions after crystallization of the SLN compositions may reflect that the drug had separated out, and this probably contributed to the decrease in the $\mathrm{EE} \%$ after 1 month of storage.

Non-formulated PK-L4 demonstrated a burst effect, which is the rapid release of the drug into the blood causing the concentration to quickly rise then briefly plateau. It causes an excessive drug concentration beyond the therapeutic range, which is commonly observed initially, followed by slow and incomplete drug release. The PK-L4 solution and PK-L4-loaded SLNs formulation showed $\mathrm{IC}_{50}$ values lower than that of the doxorubicin solution in the A549, MCF7, MDA-MB-231, and Ca9-22 cell lines. Therefore, the therapeutic index needs to be examined. Programming the release rate of a drug is an issue of concern in controlled drug-delivery systems, since a drug overdose can cause serious cytotoxicity in release regions; this is especially true of anticancer drugs. ${ }^{30}$ Theoretically, PK-L4 is water insoluble, so it can be embedded in the inner core of lipid nanoparticles, followed by its slow release to the external phase. This phenomenon results in the sustained and controlled delivery of the drug. In general, the drug was stably retained in the lipid core, and we determined the external phase in the medium determine the release rate and to calculate the release parameters. The drug release rate of PK-L4-loaded SLNs with PEG was slower than that of the group without PEG, which can be attributed to PEG chains on the nanoparticle surfaces acting as a hydrophilic shield surrounding the nanoparticles. The PK-L4 solution obviously presented Higuchiorder release parameters, and SLNs with or without PEG fit a zero-order equation. Previous research has also indicated that paclitaxel released from SLNs incorporating F-68 similarly presented zero-order kinetics. ${ }^{31}$ This phenomenon suggests that hydrophobic drugs released from SLNs followed zero order and present sustained-release kinetics. Although some of the literature has described enrichment of a drug surrounding the core shells when SLN formulations cool down, especially when using the hot homogenization process, ${ }^{32}$ the effect of burst release was not present in this case.

Conventional chemotherapeutic agents lack specificity, so an increase in the dose requirement can cause toxic side effects. ${ }^{33}$ PK-L4 was synthesized as a potential anticancer compound, and $\mathrm{IC}_{50}$ values were significantly lower than those of the doxorubicin solution in the A549, MCF7, MDAMB-231, and Ca9-22 cancer cell lines. The cytotoxicity of PK-L4-loaded SLNs with PEG monostearate significantly decreased $\mathrm{IC}_{50}$ values, particularly in the A549, MCF7, and MDA-MB-231 cell lines, which exhibited highly sensitive cytotoxicity. This evidence was strongly connected to SLNs incorporating PEG. Two important points should be highlighted at this point. First, one of the reasons for this is that the degradation of SLNs can be retarded using stabilizing surfactants. ${ }^{27}$ PEG monostearate is an amphiphilic polymeric derivative of hydrophilic PEG modified by a hydrophobic moiety, and it can be easily incorporated into the lipid core of colloidal carriers with hydrophilic PEG chains on their surface. ${ }^{34}$ Second, it is well-known that PEG that coats or modifies the surfaces of drug carriers can improve the surface hydrophilicity. Thus, the hydrophilic layer creates a barrier preventing the adsorption of lipoproteins and opsonins onto surfaces of drug carriers, thus allowing PEG-coated/modified drug carriers to circulate in the blood for longer times. ${ }^{35,36}$

The $t_{1 / 2 \alpha}$ of SLNs with PEG was higher than those of the control and the group without PEG, which indicates that the SLNs with PEG were rapidly distributed from the central compartment to tissue compartments. Moreover, the Vss and MRT also demonstrated that SLNs with PEG rapidly left the plasma. Overall, we assumed that PK-L4-loaded SLNs would be rapidly distributed from the central compartment to tissue compartments. We also calculated the relative bioavailability, which indicated significant increases in the 
kidneys and large intestine (data not shown). Actually, colloidal drug carriers are generally recognized by macrophages due to their physicochemical characteristics such as surface charge, particular size, and surface hydrophobicity. It was shown that PEG improved the surface hydrophilicity of carriers, thereby preventing the carriers recognized by the reticuloendothelial system in vivo, allowing the carriers to remain in circulation for longer times. ${ }^{37}$

Nanoparticles are PEGylated to form a hydrated stealth shell that protects particles from destruction by proteins; hence, PEG can prolong the blood circulation time of a drug. Compared to classical nanoparticles, their PEGylated counterparts show increased half-lives, decreased plasma clearance rates, and shifts in the distribution in favor of diseased tissues. ${ }^{38}$ Generally, a drug is released from the excipients by diffusion and by using endogenous enzymes that degrade the excipients (particular lipids). Hence, selecting a suitable lipid type is an important consideration in formulation design as it influences the degradation process. ${ }^{39}$

In this study, the PEGylation of the SLNs with PEG monostearate changed the biodistribution behavior of the SLNs with their PK-L4 cargo. Part of the reason was due to the size of these nanoparticles which are normally in the range of 10-200 nm; this is small enough to avoid filtration by the lungs and spleen. ${ }^{40}$ In contrast, after intravenous administration, lipid emulsions are rapidly taken up by the reticuloendothelial system in the liver and spleen. Moreover, high concentrations of free-type PK-L4 were located in the spine, which was mainly due to the lipophilicity of PK-L4. ${ }^{41}$ Past research has also revealed that the biodistribution behavior of nanoparticles can be altered by coating them with non-ionic surfactants such as polysorbates, poloxamers, and poloxamines. ${ }^{42}$ Nanoparticles coating these elements cause the apolipoprotein E of plasma to be adsorped onto the surface of the nanoparticles. These coated particles behave as low-density lipoprotein (LDL) particles and may interact with LDL receptors, initiating their uptake by endothelial cells. Subsequently, either the released drug might diffuse into brain cells or the particles may be transcytosed. Polysorbate 80 appears to act as an anchor for apolipoprotein E. ${ }^{43}$

\section{Conclusion}

We prepared PEGylated SLNs to overcome the insolubility of PK-L4 and established its physiochemical properties and biodistribution behavior. The main advantage of PEGylated SLNs is that they can successfully encapsulate hydrophobic PK-L4 in the inner core of SLNs. The size, in the range of $10-200 \mathrm{~nm}$, is small enough to avoid filtration by the liver and spleen, ensuring the potential passive targeting of tumors through an enhanced permeability and retention effect. Moreover, PK-L4-loaded PEGylated SLNs were rapidly distributed from plasma to organs. Generally, PK-L4 exhibited higher accumulation in specific spine, lung, and large intestine locations. Current research indicates that PK-L4 is a powerful anticancer drug that needs a better delivery system because it holds much promise for treating cancers in the near future; the PEGylated SLNs described here may be a suitable new delivery system for this drug.

\section{Disclosure}

The authors have no conflicts of interest to disclose.

\section{References}

1. Arlin ZA, Feldman E, Kempin S, et al. Amsacrine with high-dose cytarabine is highly effective therapy for refractory and relapsed acute lymphoblastic leukemia in adults. Blood. 1988;72(2):433-435.

2. Legha SS, Keating MJ, McCredie KB, Bodey GP, Freireich EJ. Evaluation of AMSA in previously treated patients with acute leukemia: results of therapy in 109 adults. Blood. 1982;60(2):484-490.

3. Paxton JW, Kim SN, Whitfield LR. Pharmacokinetic and toxicity scaling of the antitumor agents amsacrine and CI-921, a new analogue, in mice, rats, rabbits, dogs, and humans. Cancer Res. 1990;50(9):2692-2697.

4. Su TL, Chou TC, Kim JY, et al. 9-substituted acridine derivatives with long half-life and potent antitumor activity: synthesis and structureactivity relationships. J Med Chem. 1995;38(17):3226-3235.

5. Gamage SA, Figgitt DP, Wojcik SJ, et al. Structure-activity relationships for the antileishmanial and antitrypanosomal activities. J Med Chem. 1997;40(16):2634-2642.

6. Chen IL, Chen YL, Tzeng CC, Chen IS. Synthesis and cytotoxic evaluation of some 4-anilinofuro[2,3-b]quinoline derivatives. Helv Chim Acta. 2002;85(7):2214-2221.

7. Finlay GJ, Riou JF, Baguley BC. From amsacrine to DACA (N-[2(dimethylamino)ethyl] acridine-4-carboxamide): selectivity for topoisomerases I and II among acridine derivatives. Eur J Cancer. 1996;32A(4):708-714.

8. Zhao YL, Chen YL, Tzeng CC, Chen IL, Wang TC, Han CH. Synthesis and cytotoxic evaluation of certain 4-(phenylamino)furo[2,3-b]quinoline and 2-(furan-2-yl)-4-(phenylamino)quinoline derivatives. Chem Biodivers. 2005;2(2):205-214.

9. Chen YL, Chen IL, Wang TC, Han CH, Tzeng CC. Synthesis and anticancer evaluation of certain 4-anilinofuro[2,3-b]quinoline and 4-anilinofuro[3,2-c] quinoline derivatives. Eur J Med Chem. 2005;40(9):928-934.

10. Pegg DG, Watkins JR, Graziano MJ, McKenna MJ. Subchronic intravenous toxicity of the antineoplastic drug, amsacrine, in male Wistar rats. Fundam Appl Toxicol. 1996;32(1):45-52.

11. Kestell P, Paxton JW, Evans PC, et al. Disposition of amsacrine and its analogue 9-([2-methoxy-4-[(methylsulfonyl)amino]phenyl]amino)-N,5dimethyl-4-acridinecarboxamide (CI-921) in plasma, liver, and Lewis lung tumors in mice. Cancer Res. 1990;50(3):503-508.

12. Solid lipid nanoparticles: an effective lipid based technology for poorly water soluble drugs. Int J Pharm Sci Rev Res. 2010;5(2):78-90.

13. Hou D, Xie C, Huang K, Zhu C. The production and characteristics of solid lipid nanoparticles (SLNs). Biomaterials. 2003;24(10):1781-1785.

14. Mallipeddi R, Rohan LC. Progress in antiretroviral drug delivery using nanotechnology. Int J Nanomedicine. 2010;5:533-547.

15. Perugini P, Tomasi C, Vettor M, et al. Influence of SLN matrix modification on "in vitro" and "in vivo" nanoparticle performances. Int J Pharmacy Pharm Sci. 2010;2(3):37-42. 
16. Kostera VS, Kuks PF, Lange R, Talsma H. Particle size in parenteral fat emulsions, what are the true limitations? Int J Pharm. 1996;134(1-2): 235-238.

17. Marianecci C, Di Marzio L, Rinaldi F, Carafa M, Alhaique F. Pulmonary delivery: innovative approaches and perspectives. J Biomater Nanobiotechnol. 2011;2(5):567-575.

18. Nanjwade BK, Patel DJ, Udhani RA, Manvi FV. Functions of lipids for enhancement of oral bioavailability of poorly water-soluble drugs. Sci Pharm. 2011;79(4):705-727.

19. Yuan H, Wang LL, Du YZ, You J, Hu FQ, Zeng S. Preparation and characteristics of nanostructured lipid carriers for control-releasing progesterone by melt-emulsification. Colloids Surf B Biointerfaces. 2007;60(2):174-179.

20. Fang C, Shi B, Pei YY. Effect of MePEG molecular weight and particle size on in vitro release of tumor necrosis factor-alpha-loaded nanoparticles. Acta Pharmacol Sin. 2005;26(2):242-249.

21. Mehnert W, Mäder K. Solid lipid nanoparticles: production, characterization and applications. Adv Drug Deliv Rev. 2001;47(2-3): 165-196.

22. Freitas C, Müller RH. Effect of light and temperature on zeta potential and physical stability in solid lipid nanoparticle (SLN ${ }^{\mathrm{TM}}$ ) dispersions. Int J Pharm. 1998;168(2):221-229.

23. Hu Y, Xie J, Tong YW, Wang CH. Effect of PEG conformation and particle size on the cellular uptake efficiency of nanoparticles with the HepG2 cells. J Control Release. 2007;118(1):7-17.

24. Wan F, You J, Sun Y, et al. Studies on PEG-modified SLNs loading vinorelbine bitartrate (I): preparation and evaluation in vitro. Int $J$ Pharm. 2008;359(12):104-110.

25. Storm G, Belliot SO, Daemen T, Lasic DD. Surface modification of nanoparticles to oppose uptake by the mononuclear phagocyte system. Adv Drug Deliv Rev. 1995;17(1):31-48.

26. Romberg B, Hennink WE, Storm G. Sheddable coatings for longcirculating nanoparticles. Pharm Res. 2008;25(1):55-71.

27. Müller RH, Mäder K, Gohla S. Solid lipid nanoparticles (SLN) for controlled drug delivery - a review of the state of the art. Eur J Pharm Biopharm. 2000;50(1):161-177.

28. Freitas C, Müller RH. Correlation between long-term stability of solid lipid nanoparticles (SLN) and crystallinity of the lipid phase. Eur $J$ Pharm Biopharm. 1999;47(2):125-132.

29. Venkateswarlu V, Manjunath K. Preparation, characterization and in vitro release kinetics of clozapine solid lipid nanoparticles. J Control Release. 2004;95(3):627-638.

30. Fang YP, Lin YK, Su YH, Fang JY. Tryptanthrin-loaded nanoparticles for delivery into cultured human breast cancer cells, MCF7: the effects of solid lipid/liquid lipid ratios in the inner core. Chem Pharm Bull (Tokyo). 2011;59(2):266-271.
31. Chen DB, Yang TZ, Lu WL, Zhang Q. In vitro and in vivo study of two types of long-circulating solid lipid nanoparticles containing paclitaxel. Chem Pharm Bull (Tokyo). 2001;49(11):1444-1447.

32. Uner M, Yener G. Importance of solid lipid nanoparticles (SLN) in various administration routes and future perspectives. Int J Nanomedicine. 2007;2(3):289-300.

33. Elbayoumi TA, Torchilin VP. Enhanced cytotoxicity of monoclonal anticancer antibody 2C5-modified doxorubicin-loaded PEGylated liposomes against various tumor cell lines. Eur J Pharm Sci. 2007;32(3):159-168.

34. Shen J, Wang Y, Ping Q, Xiao Y, Huang X. Mucoadhesive effect of thiolated PEG stearate and its modified NLC for ocular drug delivery. J Control Release. 2009;137(3):217-223.

35. Cole AJ, David AE, Wang J, Galbán CJ, Yang VC. Magnetic brain tumor targeting and biodistribution of long-circulating PEG-modified, cross-linked starch-coated iron oxide nanoparticles. Biomaterials. 2011;32(26):6291-6301.

36. Yoshizawa Y, Kono Y, Ogawara K, Kimura T, Higaki K. PEG liposomalization of paclitaxel improved its in vivo disposition and anti-tumor efficacy. Int J Pharm. 2011;412(1-2):132-141.

37. Jia L, Shen J, Zhang D, et al. In vitro and in vivo evaluation of oridonin-loaded long circulating nanostructured lipid carriers. Int $J$ Biol Macromol. 2012;50(3):523-529.

38. Tsai MJ, Huang YB, Wu PC, et al. Oral apomorphine delivery from solid lipid nanoparticles with different monostearate emulsifiers: pharmacokinetic and behavioral evaluations. J Pharm Sci. 2011;100(2):547-557.

39. Fang YP, Wu PC, Tzeng CC, Chen YL, Lin HL, Tsai YH. A new antitumor agent, (3-chloro-7-methoxyfuro[2,3-b]-quinolin-4-yl)(4-methoxyphenyl), loaded in solid lipid nanoparticles: characterization and pharmacokinetics. Curr Nanosci. 2012;8(2):266-273.

40. Moghimi SM, Hunter AC, Murray JC. Long-circulating and target-specific nanoparticles: theory to practice. Pharmacol Rev. 2001;53(2):283-318.

41. Manjunath K, Venkateswarlu V. Pharmacokinetics, tissue distribution and bioavailability of clozapine solid lipid nanoparticles after intravenous and intraduodenal administration. $J$ Control Release. 2005;107(2):215-228.

42. Liu M, Li H, Luo G, Liu Q, Wang Y. Pharmacokinetics and biodistribution of surface modification polymeric nanoparticles. Arch Pharm Res. 2008;31(4):547-554.

43. André P, Komurian-Pradel F, Deforges S, et al. Characterization of low- and very-low-density hepatitis $\mathrm{C}$ virus RNA-containing particles. J Virol. 2002;76(14):6919-6928.
International Journal of Nanomedicine

\section{Publish your work in this journal}

The International Journal of Nanomedicine is an international, peerreviewed journal focusing on the application of nanotechnology in diagnostics, therapeutics, and drug delivery systems throughout the biomedical field. This journal is indexed on PubMed Central,

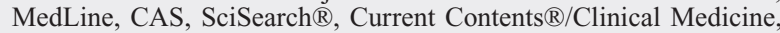

\section{Dovepress}

Journal Citation Reports/Science Edition, EMBase, Scopus and the Elsevier Bibliographic databases. The manuscript management system is completely online and includes a very quick and fair peer-review system, which is all easy to use. Visit http://www.dovepress.com/ testimonials.php to read real quotes from published authors. 